\title{
Philips Pinnacle
}

National Cancer Institute

\section{Source}

National Cancer Institute. Philips Pinnacle. NCI Thesaurus. Code C105677.

A proprietary radiation therapy planning system developed by the Philips corporation. 